\title{
Archéopages
}

Archéopages

Archéologie et société

Hors-série 2 | 2010

Archéologie sans frontières

\section{De Reqem à Pétra : investigations au cœur de la Cité rose}

François Renel, Christian Augé, Mehdi Belarbi et Xavier Peixoto

\section{OpenEdition}

1 Journals

Édition électronique

URL : https://journals.openedition.org/archeopages/816

DOI : 10.4000/archeopages.816

ISSN : 2269-9872

Éditeur

INRAP - Institut national de recherches archéologiques préventives

Édition imprimée

Date de publication : 1 octobre 2010

Pagination : 129-135

ISSN : $1622-8545$

\section{Référence électronique}

François Renel, Christian Augé, Mehdi Belarbi et Xavier Peixoto, « De Reqem à Pétra : investigations au cœur de la Cité rose », Archéopages [En ligne], Hors-série 2 | 2010, mis en ligne le 01 octobre 2010 consulté le 23 février 2023. URL : http://journals.openedition.org/archeopages/816 ; DOI : https:// doi.org/10.4000/archeopages.816 
confirmé ensuite qu'ils ne fonctionnaient pas en même temps et que le four oriental semble antérieur au four principal. La partie occidentale de l'aire de fouille a également révélé des structures inattendues. Il s'agit de plusieurs massifs alignés en bordure du mur récupéré ; ces maçonneries ont été interprétées comme des rampes facilitant l'accès à la voûte du bassin pour le déversement des matières brutes et le contrôle de la cuisson. Cette zone, très soignée, est dallée de briques, témoignant ainsi d'une aire de travail «propre» à fort passage. La densité des vestiges découverts au fur et à mesure de la fouille a contraint, en plus des objectifs définis, à travailler sur les nouvelles découvertes, imposant un rythme de travail plus soutenu.

Les orientations de l'année 2008 visaient à l'aboutissement du programme quinquennal en répondant au maximum de questions soulevées par ces structures. Ainsi, les trois semaines dédiées à l'achèvement de la fouille du secteur 2 ont visé à la compréhension des différents états de construction des structures, des modes d'utilisation de la chambre de chauffe et de l'évolution chronologique de la zone. Pour ce faire, une coupe transversale de la partie sud du secteur a été réalisée suivant un axe est-ouest. Elle a offert la totalité de la séquence stratigraphique de cette zone et permis d'appréhender les relations chronologiques entre le bassin de fusion, les rampes d'accès, l'espace dallé et, par extension, le bassin oriental. Une seconde coupe a été implantée dans la chambre de chauffe afin de comprendre le mode de construction de la bordure et du mur est de l'atelier, et de compléter les connaissances sur la chambre de chauffe, ainsi que sa séquence chronologique.

La campagne 2008 a marqué l'arrêt de la fouille du secteur 2. La phase d'étude des données de terrain a commencé en 2009 en vue d'une publication des résultats en 2012. Pour la post-fouille, il s'est avéré indispensable de terminer la base de données (qui combine unités stratigraphiques, faits, mobilier, documentation photographique et graphique). Cette base a permis d'élaborer plus facilement le diagramme stratigraphique du secteur 2. Cette tâche fut la plus longue à mettre en œuvre. L'arbre stratigraphique offre une organisation visuelle du secteur permettant par la suite de confondre unités et structures pour retracer le développement de l'atelier tout au long de sa période de fonctionnement. Ces données sont ensuite couplées aux facteurs graphiques. Dans un premier temps, les relevés en coupe ont été regroupés suivant leur proximité de localisation et leur position en altitude, afin de restituer la succession des structures et des unités stratigraphiques ; les résultats ont été ensuite comparés au diagramme pour validation.

Ainsi, cinq horizons chronologiques ont été définis, qui correspondent aux utilisations successives de l'atelier et se divisent en plusieurs phases (construction des structures, occupation ou utilisation, puis destruction ou abandon). Le report des relevés en coupe sur le plan d'ensemble permet ensuite de transposer les horizons chronologiques définis par la stratigraphie à l'ensemble des structures composant le secteur 2 . Il offre alors une vision générale du développement de l'atelier. La datation relative des unités stratigraphiques est ensuite confrontée à la datation absolue issue du mobilier. Celui-ci se résume à des fragments de céramiques communes et d'amphores qui ne peuvent être datées précisément dans la fourchette de trois siècles d'utilisation de ce secteur, en raison de l'absence de chronotypologies bien établies. Des prélèvements de cendres et de terre cendreuse ont été effectués afin de préciser la nature des combustibles employés et obtenir des datations au carbone 14 (laboratoire de l'Ifao).

\section{De Reqem à Pétra : investigations au cœur de la Cité rose}

\author{
François Renel \\ Inrap, UMR 7041 "Archéologies et Sciences de l'Antiquité», Aphor \\ Christian Augé \\ CNRS, IFPO \\ Mehdi Belarbi \\ Inrap \\ Xavier Peixoto \\ Inrap
}

$\mathrm{M}$

entionnée brièvement dans les textes aux $\mathrm{IV}^{\mathrm{e}}-\mathrm{III}^{\mathrm{e}}$ siècles avant notre ère, Reqem ou Pétra, cité caravanière et capitale d'un royaume unifié, connaît une longue histoire marquée par la royauté nabatéenne puis par l'intégration à l'Empire romain en 106 de notre ère, mettant fin à son statut d'État-client. Le tremblement de terre de 363 marque un tournant décisif dans le développement de la cité qui sombrera par la suite progressivement dans l'oubli au moment de l'avènement de l'islam. Redécouverte en 1812 par le Suisse Johann Burckhardt, la ville antique de Pétra a depuis fait l'objet de recherches scientifiques dès le milieu du XIX ${ }^{\mathrm{e}}$ siècle, poursuivies et accélérées grâce au développement de l'archéologie moderne à partir de la seconde moitié $\mathrm{du} \mathrm{Xx}^{\mathrm{e}}$ siècle. Paradoxalement, au-delà de ces grandes lignes, son histoire reste très mal connue : les sources épigraphiques demeurent quasi-absentes et les quelques textes des auteurs anciens ne retranscrivent pas, ou de façon tronquée, ce que l'archéologie met au jour depuis une vingtaine d'années.

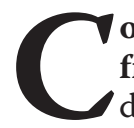
omplexité contextuelle des fouilles françaises à Pétra. Les fouilles françaises de Pétra, partie intégrante du programme «De Pétra à wadi Ramm, le sud jordanien nabatéen et arabe» (dirigé par Christian Augé), visent à étudier l'évolution d'une partie du centre de la ville antique dominée par l'implantation du sanctuaire dit «Qasr al-Bint Firaoun». Celui-ci, dédié à Dushara, dieu principal de la cité, est cerné par tout un ensemble d'édifices relatifs au culte et à sa gestion [Fig.1]. Les dégagements menés depuis 1999 montrent que, si la structuration actuelle de l'espace
1 Diodore, II, 48 et XIX 94-100. Les Nabater
sont cités comme des Bédouins nomades. 

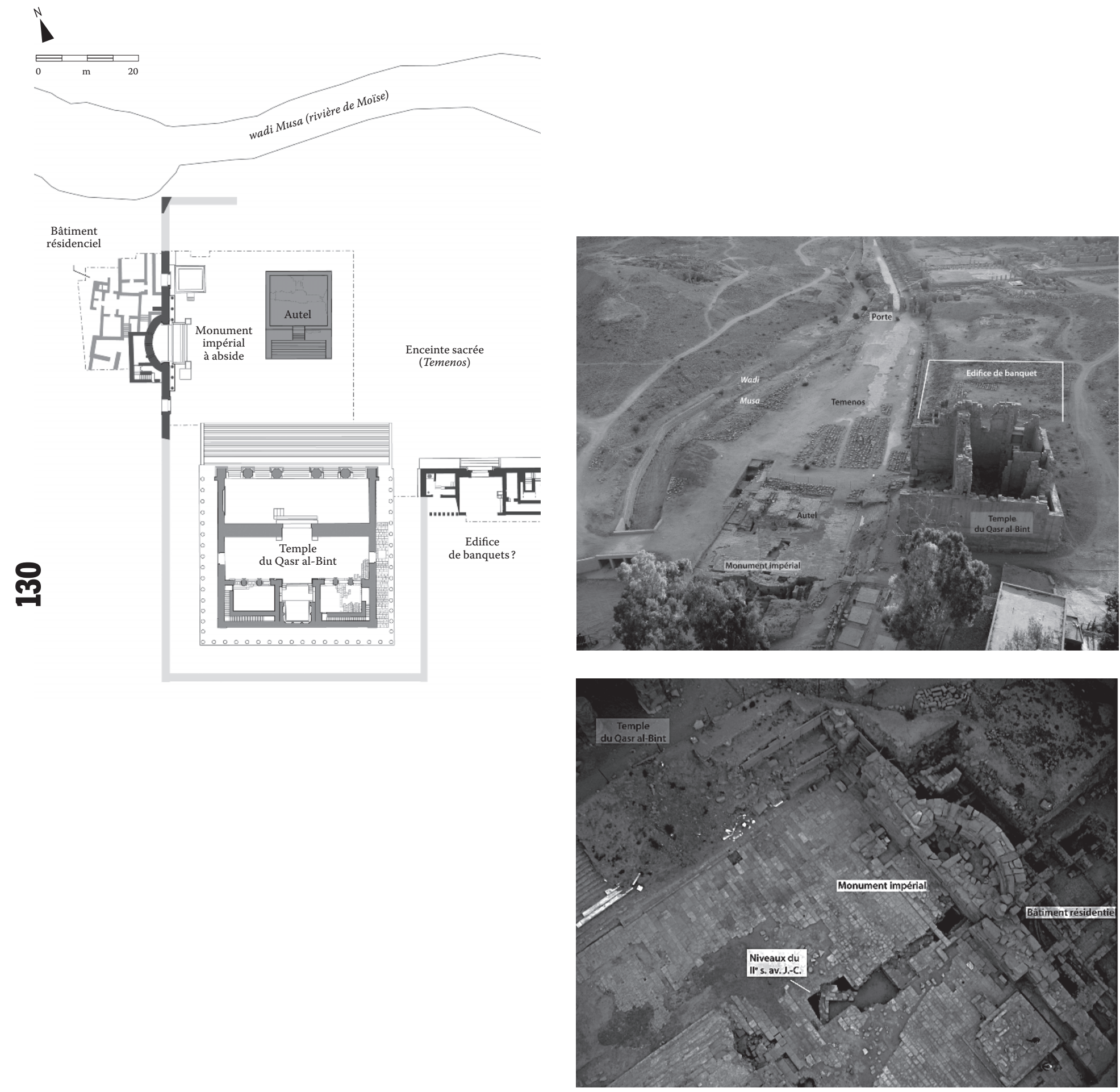

[Fig. 1] Plan général des vestiges mis au jour depuis 1999 dans la partie orientale du temenos autour du temple du Qasr al-Bint.
[Fig.2] Vue de la cuvette naturelle constituant le centre-ville antique de Pétra prise de la falaise d'El-Habis. Le temple et son temenos sont desservis par une porte

monumentale construite sous le règne de l'empereur Trajan.
[Fig.3] Cette vue de la partie occidentale du temenos montre bien la

superposition et la complexité des différentes phases architecturales et le caractère monumental du lieu.

Cliché réalisé au moyen d'un ballon sonde mis à disposition par la mission italienne de Pétra. 
remonte, pour l'essentiel, à la période nabatéenne classique ( $\mathrm{I}^{\mathrm{er}}$ siècle avant notre ère- $\mathrm{I}^{\mathrm{er}}$ siècle de notre ère), période d'apogée du site, l'occupation et l'aménagement de ce secteur de la ville s'échelonnent cependant sur une durée beaucoup plus longue. Celle-ci remonte au IV ${ }^{\mathrm{e}}$ siècle avant notre ère pour s'achever avec la période des croisades. La fouille s'y révèle particulièrement complexe en raison de l'articulation de deux vastes ensembles architecturaux en cet endroit, dont la longévité a été ponctuée par des incendies, des phases d'abandon et de destruction liées à des événements historiques et aux activités sismiques de la région.

Ce programme, initié par l'Institut français du Proche-Orient à la demande des autorités jordaniennes, présente la particularité d'intégrer une équipe d'archéologues intégralement issue de l'Inrap, la direction sur le terrain étant assurée, depuis le début du programme, par François Renel (membre de l'IfPo à cette date), avec la collaboration depuis 2002 de Mehdi Belarbi et de Xavier Peixoto, dans le cadre de son programme de conventions internationales. Cette opération, d'une durée d'un mois par an, financée par la commission des Affaires étrangères, est pluridisciplinaire avec l'association des chercheurs de l'UMR 7041 du CNRs (outre Christian Augé, Jacqueline Dentzer-Feydy assure l'étude des blocs architecturaux et Michel Mouton intervient sur les niveaux hellénistiques), et l'Institut français du ProcheOrient (architectes Laurent Borel et Chrystelle March). Les jours PAs internationaux dévolus à cette opération ont permis, à raison d'un important investissement personnel, d'encadrer sur le terrain une équipe d'ouvriers locaux et de participer activement à l'élaboration des différents projets de publication. Cet aspect s'est trouvé renforcé depuis 2008, date à partir de laquelle le programme Qasr al-Bint se poursuit en parallèle à une ANR orientée sur la période hellénistique à Pétra et tournée vers les recherches paléoenvironnementales (analyse des anciennes terrasses agricoles, calcul de la diète des troupeaux grâce à des analyses isotopiques sur les ossements et séries d'analyses au carbone 14). En marge de ces aspects institutionnels, l'équipe a pu participer à la formation d'étudiants tant français que jordaniens et assurer un rapport étroit avec la population grâce aux liens tissés sur le terrain.

Fouiller à Pétra présente de multiples particularités. Il s'agit d'un site en mouvement, se développant au gré des infrastructures touristiques et des volontés de mises en valeur successives. Ces données constituent autant de contraintes pour la réalisation des fouilles archéologiques, car les choix d'aménagements entraînent notamment une limitation spatiale et verticale de nos observations. Rappelons, dans cette problématique, que la direction des Antiquités jordanienne agit sous la tutelle du ministère du Tourisme.

À l'issue de dix campagnes, perçues par les membres de la mission comme une mise en pratique de connaissances métropolitaines et comme une acquisition de nouvelles connaissances, la fouille a livré nombre de données nouvelles qui constituent une avancée importante dans le champ d'étude du domaine nabatéen.

\section{Découvertes anascaphiques sur le} développement architectural de la Pétra antique. Topographiquement, le centre-ville antique s'établit dans une cuvette d'axe est-ouest entourée de massifs de grès rouge à rose et traversée en son milieu par le wadi Musa (rivière de Moïse). La zone d'étude est établie sur sa rive gauche au pied de la falaise dite d'El-Habis [Fig.2], siège d'une forteresse croisée implantée au XII ${ }^{\mathrm{e}}$ siècle. Elle concerne l'espace en relation avec le temenos, enceinte sacrée en relation avec le temple du Qasr al-Bint. Il s'agit d'un site urbain de grande ampleur avec une superposition de plusieurs phases d'aménagements représentant une stratigraphie cumulée parfois sur plus de $5 \mathrm{~m}$.

La réalisation de sondages menés sous le pavement de l'enceinte du sanctuaire a permis la mise au jour de l'état structuré le plus ancien connu à Pétra, antérieur au vaste programme architectural que constitue la zone des sanctuaires. Succédant à des niveaux de terrasses agricoles, un ensemble de bâtiments s'installe avec une orientation distincte des états postérieurs, associant probablement une architecture de terre sur radier de pierres à des structures plus légères (ensemble daté, par le mobilier et des datations au carbone $14, \mathrm{du} \mathrm{III}^{\mathrm{e}}$ siècle avant notre ère). Un niveau associant un silo et des trous de poteaux repérés au contact du substrat atteste le passage du nomadisme à la sédentarisation, ce qui se trouve corroboré par les écrits de Diodore de Sicile. Cette première architecture correspond historiquement à un contexte social et politique propice lié, d'une part, à la mainmise des Nabatéens sur le commerce des épices et, d'autre part, aux turbulences de la succession d'Alexandre le Grand avec l'émergence d'une royauté aux marges de son empire. Le mobilier relatif à cet habitat témoigne, en outre, de l'ouverture de ce site caravanier au grand commerce, par la présence d'amphores vinaires de Rhodes ou de Cos, de productions à vernis noir du monde égéen, de céramiques égyptiennes et probablement d'Arabie du Nord-Ouest.

À cette première phase succède, vers la fin $\mathrm{du} \mathrm{II}^{\mathrm{e}}$ ou au début du $\mathrm{I}^{\mathrm{er}}$ siècle avant notre ère, une phase architecturale beaucoup plus importante qui témoigne d'un vaste programme lié à la mise en place du sanctuaire principal sur l'ensemble de la rive gauche du wadi [Fig.3], impliquant par ailleurs la canalisation de ce dernier par d'ambitieux ouvrages hydrauliques. Le temple a fait l'objet dans les années 1980 d'une étude et d'une restauration (Zayadine et al., 2003). La fouille en cours a permis de dégager sur ses pourtours deux bâtiments dont les fonctions restent à affiner.

L'édifice qui flanque l'est du temple traduit un programme architectural d'importance, planifié de concert avec le temple lui-même. Seule une partie a pu en être dégagée. Une entrée monumentale 

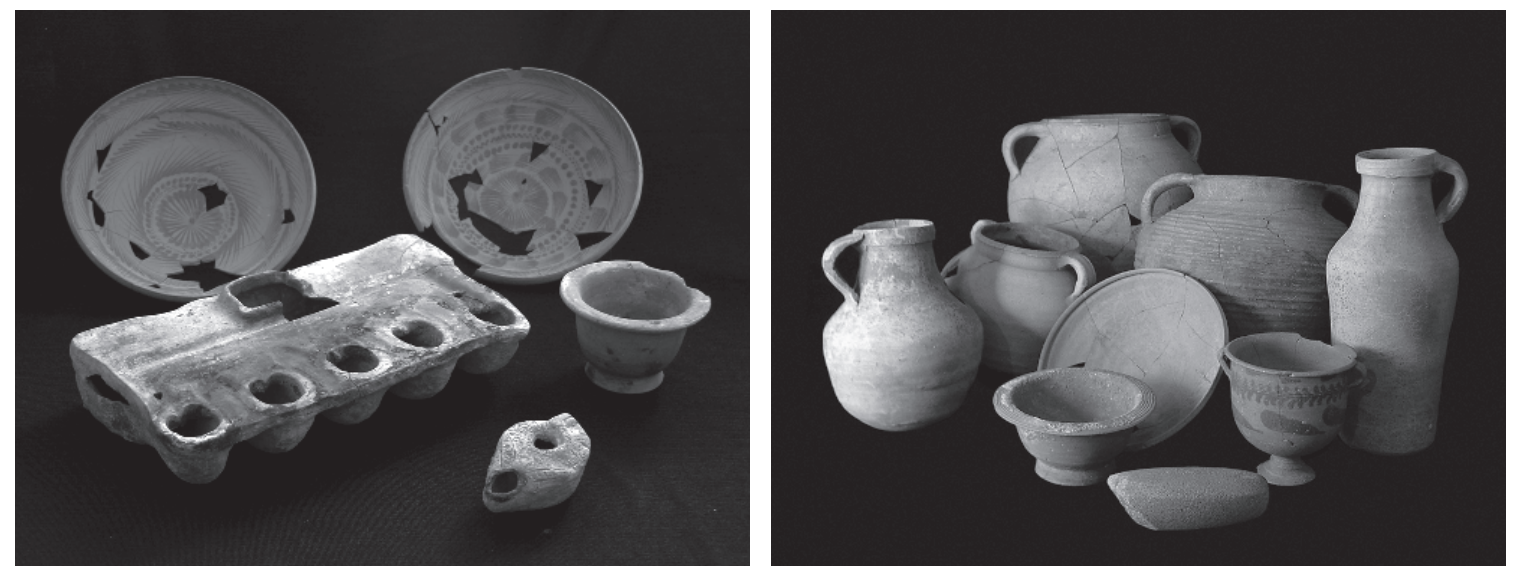

$\cong$

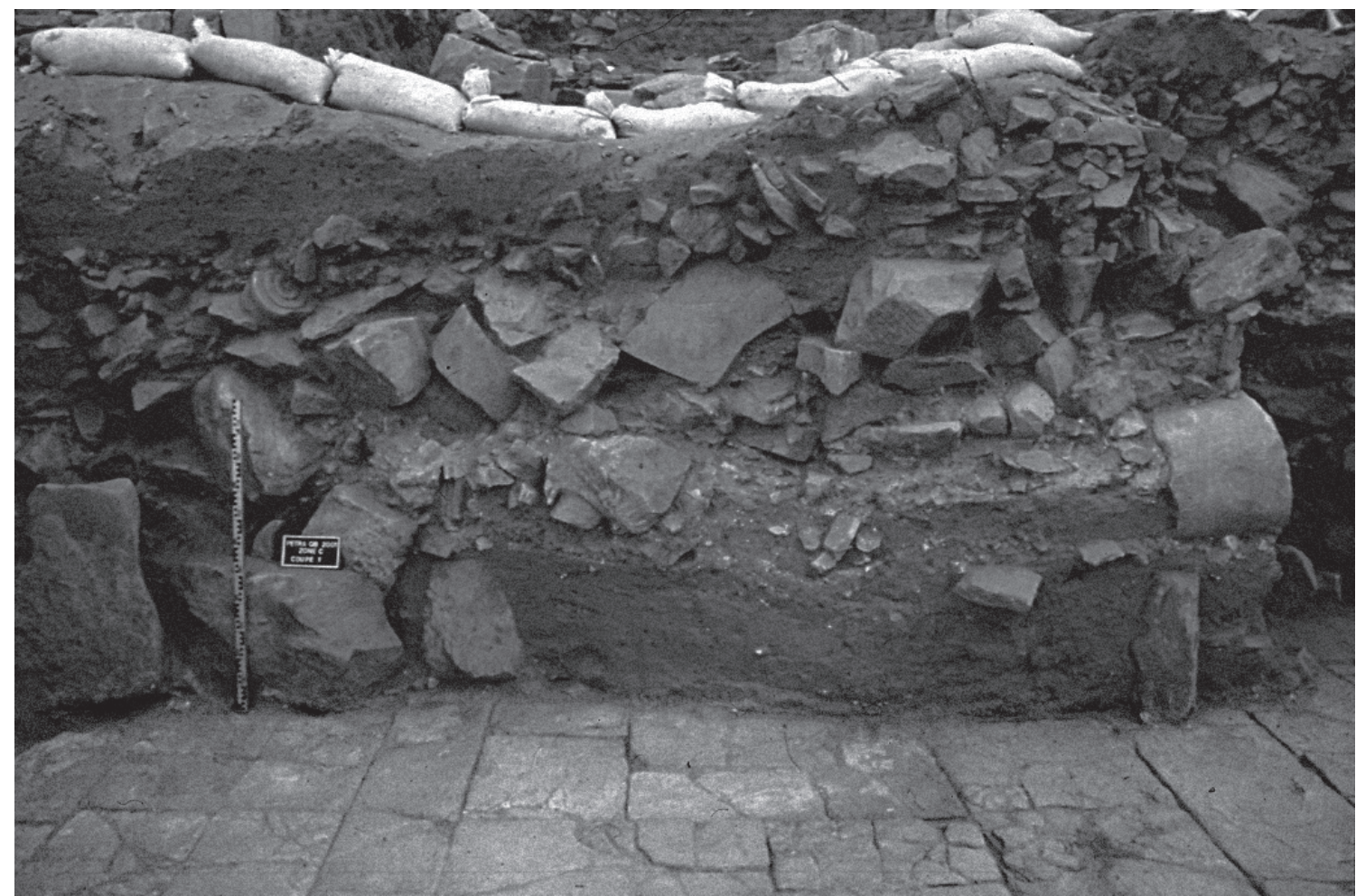

[Fig.4] Le mobilier nabatéen (fin du ${ }^{\mathrm{I}}$ s siècle avant notre ère) mis au jour dans l'édifice localisé à l'est du temple montre, aux cotés de productions fines peintes, un ensemble de lampes tabulaires de grandes dimensions liées aux banquets cultuels.
[Fig.5] Outre l'abondant matériel provenant des couches d'occupation des différents bâtiments, la fouille de la structure résidentielle située à l'ouest du monument impérial a livre, dans sa phase d'abandon, un lot de céramiques très homogènes qui servira de référence pour le domaine nabatéen.
[Fig.6] La coupe réalisée au contact du temple et du monument impérial rend compte de la succession

d'événements intervenus dans l'espace sacré à partir du III ${ }^{e}$ siècle

de notre ère, en particulier le niveau de blocs architecturaux en chute lié au tremblement de terre de 363 . 
depuis le temenos permet d'accéder à un vaste vestibule qui donnait accès à la cour proprement dite, bordée sur au moins deux côtés par une série de salles et un portique à péristyle. Bien que la cour n'ait pas encore été dégagée, une prospection radar menée en 2002 a permis d'identifier une structure centrale (petit édicule cultuel? reposoir à bétyles ${ }^{2}$ ?). L'aile nord, conservée jusqu'à l'amorce du premier étage, est constituée de pièces organisées symétriquement qui ne présentent pas d'aménagements particuliers, mais sont davantage des servitudes liées au passage, à l'eau ou à l'accès à un étage supérieur. Ce bâtiment, par sa disposition et le mobilier mis au jour dans ses niveaux d'utilisation [Fig.4], est interprété à l'heure actuelle comme un édifice pour les banquets rituels mentionnés dans les textes et liés aux confréries (thiases). Les premiers niveaux de fonctionnement de l'édifice ont livré de la céramique contemporaine du règne du roi Arétas IV et de la construction du temple.

Le bâtiment dégagé partiellement à l'ouest de la limite du temenos montre, en revanche, une organisation distincte qui manifeste davantage une extension progressive des volumes. Rasés lors de la construction de l'exèdre, les niveaux de la période nabatéenne classique, qui reposent sur un habitat antérieur, sont cependant conservés sur plus d'un mètre d'élévation. La qualité de l'habitat mis au jour, doté de cours dallées et de sols en opus sectile, traduit une architecture raffinée peut-être en rapport avec un bâtiment du clergé ou une structure palatiale. L'ensemble de ces structures connaît une première phase d'abandon au début du II $^{\mathrm{e}}$ siècle qu'il est encore difficile, dans l'état actuel de la documentation, d'associer aux effets d'un tremblement de terre daté de 114. ou à l'impact de la provincialisation, ${ }^{\mathbf{3}}$ les textes romains ne précisant pas la façon dont celle-ci s'est effectuée.

Ce passage sous la férule romaine qui coïncide avec le règne de l'empereur Trajan est perceptible par la réorganisation de l'esplanade sacrée qui aboutira, vers la fin du $\mathrm{II}^{\mathrm{e}}$ siècle, à la construction d'un vaste monument de propagande impériale mis au jour à partir de 1999. Implanté à l'ouest du temenos dont il constitue la nouvelle limite, il se présente sous la forme d'un long mur nord-sud rythmé de baies et de colonnes sur piédestaux accolés et ouvert en son centre sur une exèdre dotée d'un podium surélevé. Le mobilier provenant de sa fondation suggère un début de construction vers le tournant du $\mathrm{II}^{\mathrm{e}}$ siècle, soit peu de temps après l'intégration à la province d'Arabie. La fouille de cet édifice constitue l'un des éléments-clés de notre programme, dans la mesure où la fouille de ses abords a permis d'en comprendre la nature et d'en restituer pour partie l'élévation, avec son décor architectural, par le biais d'une anastylose des blocs retrouvés en chute. Le point d'orgue de cette étude fut la découverte en 2004 de la tête monumentale en marbre de l'empereur romain Marc Aurèle [Fig.7] qui ornait, aux côtés de la statue monumentale de Lucius Verus, l'abside achevée, comme le confirme la découverte de plusieurs inscriptions au pied du monument, sous le règne commun des deux empereurs entre 161 et 169.
Le tremblement de terre de 363 et la fin du paganisme à Pétra : traces archéologiques. La stratigraphie manifeste l'impact du tremblement de terre de 363 sur le tissu urbain (en particulier dans ce secteur de la ville), après une période de décroissance de la cité au profit de sa rivale syrienne Palmyre. Les dégagements de grande ampleur ont montré que le temple et le monument impérial ne s'étaient pas relevés de ce cataclysme.

La réutilisation de l'espace à l'issue de cet événement constitue une de nos problématiques actuelles. On observe un nettoyage partiel de l'espace encombré de décombres pour construire un habitat en place du podium destiné à la statuaire impériale. Celui-ci témoigne d'un réinvestissement des lieux, probablement désacralisés, à des fins domestiques. La présence d'objets de cultes et de bétyles révèle une persistance du paganisme dans la cité jusqu'au début du $\mathrm{V}^{\mathrm{e}}$ siècle. Ces installations construites sur les ruines du monument impérial ont, outre l'importance du corpus céramique [Fig.8] mis au jour, permis d'étudier un atelier de tabletterie et de mettre en évidence la continuation, malgré la perte de pouvoir de la cité, des grands réseaux commerciaux.

La phase la plus tardive identifiée peut être confrontée à des données historiques concernant un aspect encore peu connu de l'histoire de la Pétra byzantine : la réduction et la destruction des derniers foyers de paganisme par le christianisme triomphant à l'issue de la promulgation de l'édit de l'empereur Théodose (392) interdisant les cultes païens. La découverte, sur les secteurs fouillés, d'un important niveau cendreux daté du début du $\mathrm{v}^{\mathrm{e}}$ siècle auquel sont associées des pièces d'armement (pointes de flèche, fragments de cuirasses et éléments d'archerie en os) et des traces manifestes de pillage et de destruction, trouve peut-être son explication dans un événement historique mentionné textuellement pour cette période : la venue du moine nestorien Barsauma en 419-423 à Pétra, qui lui refuse l'entrée. Celui-ci finira par convertir les gens en masse et détruire leurs idoles. Les vestiges mis au jour dans notre complexe peuvent être le témoignage matériel de cette conversion «forcée» dans un contexte historique et politique bien documenté. Le secteur est ensuite abandonné, la ville byzantine se rétractant vers les tombeaux royaux. La construction d'un complexe ecclésiastique sur la rive droite du wadi en marque probablement la limite.

La dernière occupation perceptible de ce secteur est un espace funéraire qui se développe au milieu des édifices laissés à l'abandon. Treize sépultures enterrées en pleine terre sous une couverture en bâtière ont ainsi été mises au jour. Elles témoignent d'une fréquentation de cet espace de la ville dans les premiers temps de la conquête arabe à une date où la cité de Pétra n'est plus qu'un souvenir.

Cette première opération de fouilles françaises à Pétra est menée, en collaboration avec le personnel local des Antiquités (dont un représentant nous assiste à chaque mission), par une équipe rodée à l'archéologie urbaine employant les techniques 

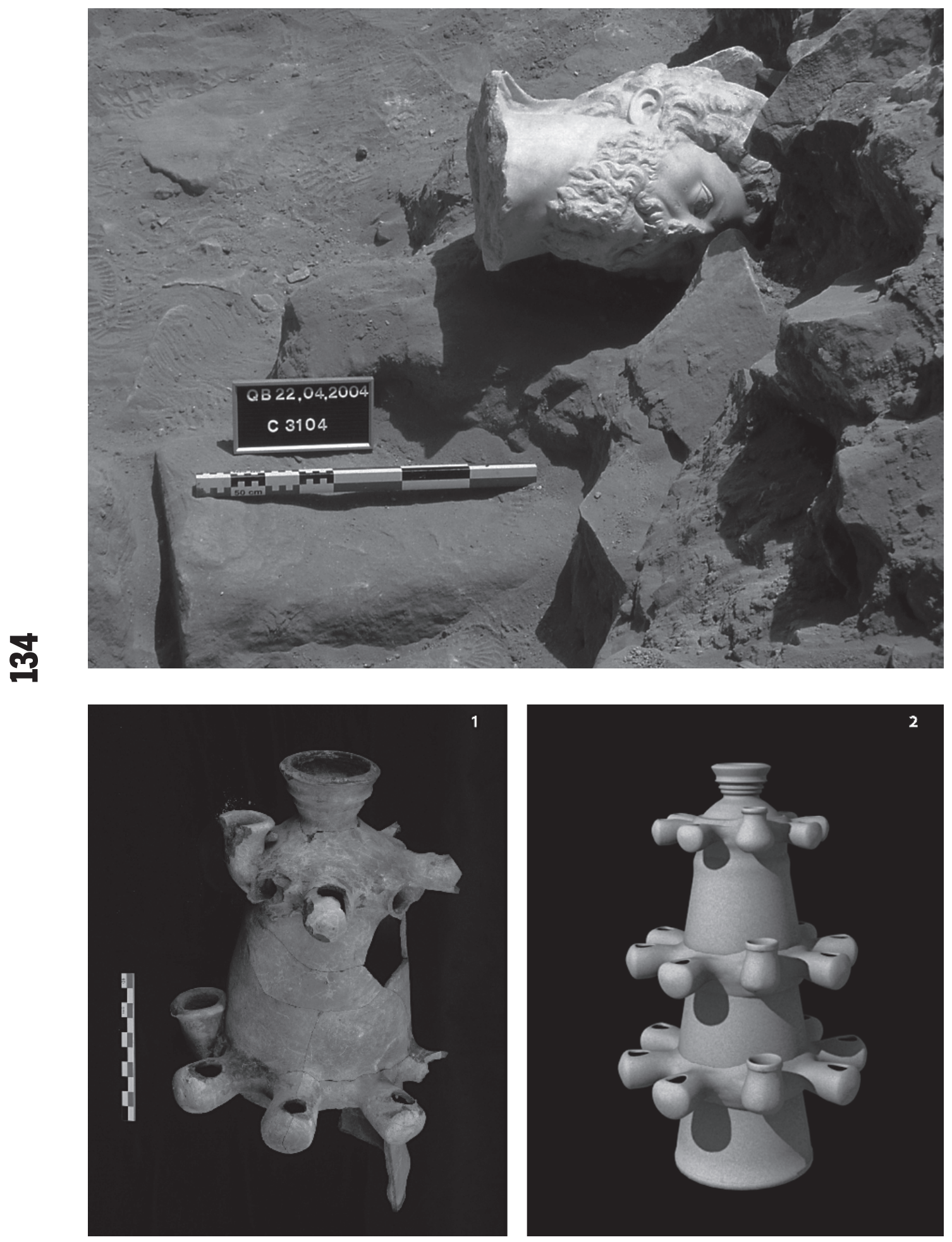

[Fig. 7] ] La découverte, en 2004, d'une tête en marbre attribuable à

l'empereur Marc Aurèle a permis

de confirmer la destination impériale du monument. Cet objet exceptionnel a fait l'objet, en 2007, d'une exposition temporaire au musée du Louvre. Elle figure désormais au Musée national à Amman.
[Fig.8] La découverte, dans le niveau d'incendie du début du $\mathrm{v}^{\mathrm{e}}$ siècle de lampes-tours inédites (1) associées à des bétyles témoigne de la poursuite des cultes païens après l'abandon du monument impérial. Leur reconstitution tridimensionnelle (2) associée à une animation permet d'en visualiser le fonctionnement. 
d'une fouille archéologique préventive étant donné les délais de la mission sur le terrain. Il présente par ailleurs, au vu de la qualité et de la diversité des vestiges, un extraordinaire champ expérimental où nous pouvons transposer l'expérience acquise en France sur des sites à large éventail chronologique et en tester la validité, ce qui témoigne de la capacité d'adaptation acquise au cours des fouilles préventives. L'archéologie en France et l'archéologie française à l'étranger sont effectivement méthodologiquement étroitement liées, comme l'a mis en exergue le regretté Serge Cleuziou. En outre, ces délais impliquent une gestion synchrone de l'acquisition des données sur le terrain et de l'étude en laboratoire, l'ensemble de la documentation devant être exploitée à l'issue des fouilles. La gestion d'une opération de ce type se trouve confrontée à un certain nombre de particularités locales qui représentent autant d'adaptations de nos techniques et de nos modes d'acquisition des données. Par exemple, à la différence des chantiers métropolitains, le terrassement se fait uniquement de façon manuelle en raison du contexte touristique, car l'usage d'engins mécanisés est entaché, dans les mentalités locales et dans celles de nombreux touristes, d'une image de destruction qui porte ombrage à la crédibilité scientifique.

Ce site, classé au Patrimoine mondial de l'humanité par l'Unesco, constitue, avec Jerash et Khirbet edh-Dharih, une vitrine de l'archéologie française en Jordanie qui accueille chaque année un nombre croissant de visiteurs. Soutenu par le ministère des Affaires étrangères et l'ambassade de France en Jordanie, notre mission a déjà connu de nombreuses visites protocolaires et servi de support à plusieurs opérations de valorisation et de communication. Dans ce cadre, elle constitue une opération prestigieuse pour l'Inrap pour valoriser nos compétences sur la scène internationale. Les années à venir s'orientent, en parallèle aux opérations de terrain, vers la publication et la valorisation des premiers résultats, en premier lieu le monument impérial à abside, en associant une étude architecturale à l'ensemble de son environnement archéologique.

Augé C., 2005, « De Pétra au wadi Ramm : le Sud jordanien nabatéen et arabe ", in Archéologies. 20 ans de recherches françaises dans le monde, ministère des Affaires étrangères, Paris, Maisonneuve et Larose, ADPF-ERC, p. 428-4.29.

Auge C., Renel F., Borel L., Dentzer-Feydy J., March C. et al., 2008 Le Sanctuaire du Qasr al-Bint à Pétra : recherches de la mission française (1999-2006), Beyrouth, IFPo.

1 Centre de recherche français à Jérusalem. Mouton M., Renel F., Kropp A., 2008, The Hellenistic levels under

\section{La mission Beisamoun : sur les traces des premiers paysans de la vallée du Jourdain}

\author{
Nicolas Samuelian \\ Inrap, DIR CIF \\ Fanny Bocquentin \\ CNRS, UMR 7041 "Archéologies et Sciences de l'Antiquité » \\ Hamoudi Khalaily \\ Israel Antiquities Authority
}

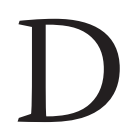
écouverte et protection du site de Beisamoun. Le site de Beisamoun est situé en Haute-Galilée (Israël), dans la partie la plus septentrionale de la vallée du Jourdain. Il faisait face à la zone marécageuse d'un grand lac, le Houleh, dont la rive la plus proche était située à 2-3 km plus au sud. L'occupation principale est datée du Néolithique précéramique B, à la fin du VIII ${ }^{\mathrm{e}}$ millénaire avant notre ère. C'est à la faveur du creusement de grands bassins piscicoles que le site de Beisamoun a été découvert, dès 1955, grâce aux prospections de surface menées par Amnon Assaf, conservateur du musée de Préhistoire du Houleh (Perrot, 1966). En 1965, 1969 et 1971, au gré des opérations de vidange et de nettoyage des bassins qui se succèdent, une équipe, dirigée par J. Perrot (CNRS/CRFJ') puis M. Lechevallier (CNRS/CRFJ), procède au relevé des structures qui émergent au fond des bassins. Associé à la collecte de matériel en surface, ce travail de repérage systématique des structures permet d'évaluer l'étendue du site néolithique à plus de 10 ha. Les bassins étant creusés en cuvette, c'est toujours sur leurs marges que les vestiges archéologiques en place ont été trouvés, ceux situés plus au centre ayant été généralement arasés. En 1972, une courte opération de sauvetage dirigée par M. Lechevallier met au jour les vestiges d'une maison rectangulaire à sol enduit (Lechevallier, 1978). Une surface d'environ $70 \mathrm{~m}^{2}$ est exposée et fouillée sur $50 \mathrm{~cm}$ de profondeur où trois niveaux d'occupation sont identifiés. Ce secteur du site a livré des informations de grand intérêt à travers, notamment, des structures variées et bien conservées, un agencement de l'espace très structuré, un outillage exceptionnel, de nombreuses inhumations et un dépôt de deux crânes surmodelés. Ces découvertes, associées à l'importante superficie du village, ont fait de Beisamoun un site majeur de la fin du Néolithique précéramique.

Par la suite, les activités agricole et piscicole ont the Temenos of the Qasr al-Bint at Petra, Annual of the Department of Archaeology of Jordan, vol. 52.

Zayadine F., Larché F., Dentzer-Feydy J., 2003, Le Qasr al-Bint de Pétra. L'architecture, le décor, la chronologie et les dieux, Paris, Adpf-Erc. jusqu'à ce quel'Office Iffice des antiquités israéliennes le protège en 1999. En 2007 et 2008, notre équipe a mené deux courtes campagnes exploratoires, de 12 jours chacune, qui ont permis de localiser un vaste secteur bien préservé du site (Bocquentin et al., 2007) et de poser les jalons d'une recherche programmée pluridisciplinaire bénéficiant des savoir-faire spécifiques des différents membres de l'équipe. En 2009 et 2010 nous avons mené des campagnes de trois semaines qui ont permis d'élargir l'emprise de fouille à $270 \mathrm{~m}^{2}$. Une quinzaine d'étudiants bénévoles participent 